\title{
Variations
}

Variations

Revue internationale de théorie critique

22 | 2019

Gorz, l'intempestif

\section{Une lecture adornienne des séries télévisées}

\section{David Buxton}

\section{(2) OpenEdition}

Journals

Édition électronique

URL : http://journals.openedition.org/variations/1149

DOI : 10.4000/variations. 1149

ISSN : 1968-3960

Éditeur

Les amis de Variations

Référence électronique

David Buxton, «Une lecture adornienne des séries télévisées », Variations [En ligne], 22 | 2019, mis en ligne le 04 mars 2019, consulté le 10 décembre 2020. URL : http://journals.openedition.org/variations/ 1149 ; DOI : https://doi.org/10.4000/variations.1149

Ce document a été généré automatiquement le 10 décembre 2020.

Les ami•e•s de Variations 


\title{
Une lecture adornienne des séries télévisées
}

\author{
David Buxton
}

1 S'agissant de l'analyse des séries télévisées, y aurait-il un intérêt à revenir sur les quelques textes courts écrits par Theodor W. Adorno sur la télévision, passablement oubliés en raison de leur statut objectivement daté? En effet, Adorno écrit sur un médium encore émergeant dont le modèle économique et les formes endogènes ne sont pas encore fixés. Si on veut rendre justice à l'article publié aux États-Unis en 1954 (Adorno, 1990), et accouché dans un contexte très difficile pour la pensée critique, on ne peut se contenter d'une glose académique. Soit on écarte du canon adornien ce texte, qui est très loin d'avoir la force du célèbre chapitre sur la Kulturindustrie, comme un produit des circonstances, soit on le fait subir à un travail critique, afin de mieux avancer dans l'esprit d'Adorno, ce qui est mon vrai point de départ. Je ne cherche pas donc à "sauver " son analyse, déjà périmée de toute façon, mais de remettre en jeu certains aspects de celle-ci dans un tout autre contexte où, espérons-le, « la pensée critique n'a pas dit son dernier mot $»^{1}$.

2 Lors d'un retour à contrecœur aux États-Unis pendant dix mois (1952-3), Adorno, sollicité, devient directeur scientifique de la Fondation Hacker, poste qui, pour prestigieux qu'il soit en apparence, le condamne en fait à mener pratiquement seul des recherches empiriques de faible ampleur ${ }^{2}$. Dans le sillage de l'étude classique sur la personnalité autoritaire menée collectivement à la fin des années 1940, Adorno prétend étudier «l'effet potentiel de la télévision - son impact sur les couches diverses de la personnalité ", problématique enracinée dans la psychologie des profondeurs ${ }^{3}$, qui tire vers une forme d'intervention pédagogique peu compatible avec la théorie critique ${ }^{4}$. Ce choix pragmatique peut s'expliquer en grande partie par le contexte politique délétère de l'époque (le maccarthysme), qui enlevait toute légitimité à des concepts politiquement chargés comme «idéologie $»^{5}$. Mais ce terme apparaît explicitement dans le titre de la version allemande de son travail, «Fernsehen als Idéologie » (Adorno, 2003b) ${ }^{6}$. Compte tenu de sa mention pléthorique et très cohérente dans la version allemande, il est raisonnable de supposer que la notion d'idéologie soit aussi présente en filigrane dans le texte 
américain de 1954. Dans un article ultérieur sur son expérience américaine, Adorno dit de ce texte qu'il fait partie de ses « recherches sur l'idéologie » (Adorno, 2003c : 296).

L'autre facteur contraignant vient du programme de la fondation Hacker, extension d'une clinique fondée en 1945 à Los Angeles par le psychiatre et psychanalyste d'origine autrichienne, Frederick (Friedrich) Hacker, qui a par la suite témoigné comme expert devant un sous-comité du Sénat sur le problème de la délinquance juvénile. Hacker était de l'avis que regarder des films violents pourrait activer des tendances latentes à des comportements agressifs. Adorno ne suit pas Hacker sur ce terrain-là, mais son approche reste encadrée par la problématique des effets de la télévision sur le comportement social, alors que la venue de la télévision devait prolonger, voire radicaliser l'analyse qu'il a déjà effectuée de l'industrie de la culture, en particulier du cinéma, dans La Dialectique de la Raison. Une ambiguïté importante parcourt inéluctablement l'étude d'Adorno entre une problématique empirique des effets, et une problématique théorique de l'idéologie.

\section{L'analyse de la télévision d'Adorno}

4 Dans cet article américain, « La télévision et les patterns de la culture de masse » (1954) ${ }^{7}$, l'analyse concrète s'appuie sur trois fictions dont une seule est identifiable, «Dante's Inferno » (CBS, 1952), qui se révèle être le premier des huit épisodes, tous écrits par le futur cinéaste Blake Edwards (notamment de la série des Panthère rose), d'un cycle interne de l'anthologie Four Seasons Playhouse diffusée entre 1952 et 1956. Le cycle est fondé sur le personnage de Willie Dante, ancien gangster réformé et propriétaire d'une boîte de nuit qui abrite un casino illégal dans une arrière-salle. Il s'agit donc d'un cycle faiblement sériel à mi-chemin entre l'anthologie et la semi-anthologie, formes historiquement situées qui influent nécessairement sur les conclusions générales tirées par Adorno.

5 Pendant les années 1950, la sérialité télévisuelle a généralement pris la forme de l'anthologie (même contenu générique, personnages et intrigues différents pour chaque épisode) ou de la semi-anthologie (un ou plusieurs personnages récurrents, intrigues et thèmes variés) ${ }^{8}$. L'épisode étudié par Adorno relève d'une variante de l'anthologie: le cycle de comédiens vedettes jouant des rôles différents par rotation, tantôt dans une veine comique, tantôt dans une veine dramatique ${ }^{9}$. Il s'agit d'une récurrence fondée sur les mêmes comédiens, et non sur les mêmes personnages, celui (faiblement) récurrent (8 fois dans 130 épisodes) de Willie Dante étant le seul de toute l'anthologie. Le personnage sériel de Dante esquisse donc celui de la série semi-anthologique, la forme classique de la série proprement dite, qui a dominé dans la deuxième moitié des années 1950 lorsque les studios hollywoodiens commencent à produire pour la télévision, transformant le film de série $\mathrm{B}$ d'une heure en série télévisée de la même durée. La sérialité se limite à un ou plusieurs personnages récurrents et un même décor/lieu, permettant une grande variété d'adaptations, même si celles-ci étaient généralement limitées au genre western, afin de bénéficier des infrastructures (un ranch, un saloon, une rue principale, etc.) déjà amorties par l'industrie cinématographique ${ }^{10}$.

6 Mais alors que la diffusion du premier épisode du cycle Dante date du 9 octobre 1952, celle du deuxième ("The Squeeze ») date du 1 octobre 1953, après le retour d'Adorno en Allemagne. Autrement dit, Adorno écrit sur un exemple embryonnaire de la série télévisée, en ignorant sa future sérialité. Celle-ci n'est donc qu'immanente à son analyse. Citons un passage in extenso : 
"Quand un spectacle télévisé porte le titre "L’Enfer de Dante", quand le premier plan s'ouvre sur une boîte de nuit portant le même nom, et quand nous voyons, assis au bar, un homme à chapeau et, quelques mètres plus loin, une femme a l'air triste, outrageusement maquillée et commandant encore un verre, nous pouvons être presque certains que quelque meurtre va être commis d'ici peu. Cette situation apparemment singulière ne fonctionne à vrai dire que comme un signal qui oriente nos attentes dans une direction bien précise. Si nous n'avions précédemment vu rien d'autre que "L’Enfer de Dante", nous n'aurions pas été sûrs de ce qui allait se passer ; mais, en l'occurrence, nous sommes effectivement amenés à comprendre par des artifices à la fois habiles et maladroits que c'est un film policier, que nous sommes en droit de nous attendre à quelques actes de violence sinistres, et probablement affreux et sadiques, à ce que le héros soit sauvé d'une situation de laquelle il ne pouvait guère espérer être sauvé, à ce que la femme assise au bar ne soit pas sans doute la principale criminelle, mais à ce que la femme soit probablement en train de gâcher sa vie en étant la nana d'un gangster, et ainsi de suite. Ce conditionnement à de tels schémas universels, cependant, ne s'arrête guère au poste de télévision. La manière dont le spectateur est conduit à regarder des détails apparemment quotidiens, comme une boîte de nuit, et à considérer des éléments banals, comme des indices d'un crime possible, l'amène à regarder la vie comme si elle, et les conflits qui la remplissent pouvaient être compris en ces termes. » (Adorno, 1990 : 235-236).

7 Voilà un bon exemple de ce qui est exaspérant chez Adorno pour ses critiques, surtout dans ses analyses de la culture populaire : une très grande distance par rapport à son objet, qui lui permet de discourir sur un médium entier à partir de trois fictions qu'il ne daigne même pas (ou à peine) identifier. Il va sans dire qu'on ne pourrait pas écrire sur les séries télévisées de cette façon aujourd'hui, alors que celles-ci sont désormais saisies par le discours universitaire, qui les ont intégrées dans la grande culture, à l'instar des films de genre, des musiques "urbaines», des bandes dessinées, des jeux vidéo. Si les études des séries particulières menées sous la bannière des cultural studies manquent de distance critique par rapport à l'objet d'étude, elles ont du moins fait preuve en général d'une maîtrise locale de celui-ci.

8 C'est justement dans la restitution des détails que l'analyse d'Adorno est problématique. Grâce à YouTube, on peut désormais visionner l'épisode en question ${ }^{11}$. Or, force est à constater que la description d'Adorno est approximative et même par moments fausse ${ }^{12}$. L'homme à chapeau n'en porte pas un; la femme maquillée ne se trouve pas à quelques mètres de lui, mais fait son apparition dans la salle, sans lui, quelques séquences plus loin. C'est le barman qui fait remarquer à Dante que la femme maquillée est triste ; c'est ellemême qui informe Dante qu'elle sera prochainement assassinée, ce qui s'avéra être un stratagème. Autant pour Adorno et son intuition... Quand elle n'est pas fausse, la description d'Adorno est vague au point de se confiner aux clichés communs à tous les films de série noire, et elle n'échappe pas à quelques jugements purement subjectifs (" outrageusement maquillée», "affreux et sadiques»); symptomatiquement, Adorno parle d'un «film policier», et non d'une dramatique télévisuelle, comme si le nouveau médium n'était pas (encore) capable de sécréter ses propres formes.

Un visionnement de quelques autres épisodes survivants du cycle Dante sur YouTube permet de voir une spécificité qu'Adorno, faute de distance historique, n'a pas pu saisir. De la centaine des personnages spécifiques créés par l'anthologie Four Seasons Playhouse entre 1952 et 1956, seul Willie Dante a accédé au statut de personnage (faiblement) récurrent. L'épisode vu par Adorno ne présage en rien cette évolution: dans une fin extrêmement elliptique, Dante retrouve sans mal son établissement après avoir tué dans 
le pur style Far West le commanditaire de l'assassinat ${ }^{13}$, mais il semble qu'entre-temps son casino illicite ait été fermé par les autorités, ce qui couperait net les possibilités de tout prolongement de la situation du départ (mais dans le prochain épisode, incroyablement, le casino dans l'arrière-salle est de nouveau ouvert, comme si de rien n'était). Si l'on excepte les personnages récurrents dans les séries radiophoniques, transposés tels quels à l'écran, on assiste-là à la création endogène et sui generis du personnage récurrent à la télévision, qui permet la remise en jeu de celui-ci. Si Dante bénéficie de la sorte, c'est qu'il se prête idéologiquement au jeu.

On est frappé maintenant par l'importance dans la première décennie de la télévision américaine des personnages qui mettent à l'épreuve les normes dominantes en matière de comportement. Le personnage de Dante est manifestement inspiré par celui de Rick Blaine (Humphrey Bogart) dans Casablanca (1942), qui se trouve (légèrement) du mauvais côté de la loi, mais qui a bon fond. Ce type de personnage a été pléthorique dans les fictions sérielles télévisées des années 1950 (citons le détective privé Peter Gunn (Blake Edwards encore) ${ }^{14}$, et les braves frères Maverick, joueurs de cartes coquins et (un peu) tricheurs, dans les séries du même nom). Cela, en plus du lieu fixe stratégique (boîte de nuit, bar, casino, saloon, club de jazz), permet de situer ces fictions sérielles comme des expériences en matière de mœurs, des théâtres moraux qui exploraient de nouvelles normes de comportement pour la société de consommation de masse naissante, le plus souvent à travers le prisme distancié de la ville frontière, ultime lieu simplifié, circonscrit et idéologiquement aménagé.

Dans un autre texte sur la télévision (Adorno, 2003a), publié en allemand en 1953, Adorno s'écarte explicitement de toute lecture en termes d'effets :

«[M]ême si les techniques les plus élaborées de la recherche sociale empirique permettent d'isoler les "facteurs" propres à la télévision, ces facteurs ne reçoivent leur efficacité que dans la totalité du système. Au lieu de subir une transformation, les individus demeurent inéluctablement eux-mêmes. Il semble que la télévision fasse d'eux une fois de plus ce qu'ils sont déjà, mais en renforçant encore ce qu'ils sont. [...] Les personnes interrogées ne sont pas en mesure de formuler les effets qu'elles subissent de façon préconsciente ou inconsciente. Elles se contenteront de rationaliser, ou de déclarer vaguement que la télévision est pour elles un divertissement. » (Adorno, 2003a : 62, 67).

La notion de renforcement par rapport à «la totalité du système » remplace celle des effets isolables du médium, à l'encontre donc du projet de la Fondation Hacker, et de l'objet manifeste de l'article américain d'Adorno, même si celui-ci y parle des « diverses couches de significations superposées » que constituent les médias de masse (Adorno, 1990 : 232). Intervenant sur la question du rapport entre les produits culturels et la politique, le critique canadien John 0 . Thompson imagine, dans une démonstration antipositiviste à l'absurde, un engin fantastique qu'il appelle « l'effetomètre ", capable de mesurer les effets (politiques) d'une œuvre sur des individus (Thompson, 1993 : 285-303). Mais œuvre par œuvre, individu par individu, l'aiguille de l'effetomètre reste désespérément immobile, car les fictions diffusées à la télévision n'ont jamais eu la capacité (ni l'intention) de manipuler les individus de quelque manière que ce soit, malgré les efforts des psychologues (dont Hacker) des années 1950 d'établir un lien de causalité directe, néfaste, entre la violence vue à la télévision et les actes de violence commis réellement. En dehors du contexte américain de son étude, Adorno parle de « renforcement » de ce que les consommateurs sont déjà, ce qui présuppose une pensée conformiste déjà massivement présente, dans d'autres fictions, dans d'autres formes 
culturelles, et dans le discours courant. Appelons celle-ci, comme le fait Adorno par ailleurs, "idéologie». Si influence de celle-ci il y a, elle est diffuse et largement inconsciente; elle se rapporte non pas à une œuvre singulière, mais à la totalité de discours sociaux.

\section{Les écueils d'un traitement monographique}

Cela a plusieurs conséquences pour l'étude des fictions sérielles. D'abord, il implique que la dimension idéologique se saisit dans l'intertextualité, dans l'analyse comparative de séries différentes, non seulement entre elles, mais avec d'autres formes culturelles, et avec d'autres niveaux de discours qui irriguent le corps social. Deuxièmement, il tend à discréditer l'approche en termes de réception, dès lors qu'on suppose que l'impact d'une série est idéologique (et non comportemental), et à ce titre, largement inconscient ${ }^{15}$. Dans le cas des séries, produits historiquement assez standardisés, on peut difficilement se fixer sur des exemples d'épisodes qui se détachent des autres, à la différence de la littérature et du cinéma, qui ont réussi à établir un « canon » de grands textes, maintenus en vie dans les cursus universitaires pour leurs qualités esthétiques et historiques, ou jugées telles. Il est, en effet, difficile d'intégrer les séries dans les cursus universitaires en raison du nombre « industriel » des épisodes, et le fait que l'intérêt du public, même pour une série à grand succès, s'essouffle rapidement après sa disparition de l'antenne. La tendance actuelle dans l'académie de privilégier les séries qui «se prêtent au jeu» (en France, on a presque plus écrit sur The Wire, série très atypique, que sur toutes les autres séries réunies) n'est pas satisfaisante non plus.

Cette façon d'aborder les séries n'est pas illégitime en elle-même, mais elle prête le flanc aux accusations d'opportunisme, surtout dans le cas de la philosophie qui se déploie avec un discours tout fait (expliquer tel thème en prenant telle série comme exemple). On s'expose aussi à la tautologie de l'analyse «culturaliste » où, plutôt que de voir la série comme une construction idéologique, on prétendrait déduire des vérités sur la société américaine (la série est vue comme un « miroir » par Martin Winckler, 2002) à partir de l'un de ses artéfacts.

15 Forcément, le problème de la représentativité idéologique des séries choisies, sans parler des épisodes particuliers, se pose. Aborder les séries au prisme d'un rattachement disciplinaire (civilisation américaine, sociologie, cinéma, philosophe, sciences politiques, géographie, droit) accentue la tendance à se pencher sur les séries jugées "pertinentes ", non seulement par rapport à une discipline, mais aussi, au sein de la nébuleuse des cultural studies, par rapport à une approche théorique (sémiologie, gender studies, queer studies, etc.), ou à de grands penseurs (Foucault, Judith Butler, etc.) qui servent de patères. L'analyse de la série devient un faire-valoir disciplinaire, un outil pédagogique.

En 2017, 487 séries (contre 210 en 2009) ont été diffusées aux États-Unis sur divers supports. Seule une petite minorité des séries réussit à se prolonger après une saison. À la différence des études littéraires ou filmiques, où l'on peut toujours espérer intégrer avec de bons arguments une œuvre "mineure » dans le canon, l'étude des séries, produits culturels très industriels, se justifient en termes de leur statut supputé de "phénomène social ». La tendance actuelle dans le monde anglo-saxon est de publier des livres collectifs mobilisant une variété d'angles et d'approches pendant les premières saisons d'une série à succès, ce qui reflète les normes commerciales (et opportunistes) de plus en plus 
présentes dans l'édition universitaire ; la rapidité de ces productions milite contre la prise de distance qui permettrait à ces analyses de faire date. À cela s'ajoute le fait que les séries oubliées ne font pas un objet d'étude viable. L'universitaire s'oblige à devenir avocat de son choix, ce qui rend difficile une démarche franchement critique; une intervention réussie passe par la complicité du lectorat ou de l'audience (à qui on ne demanderait pas à s'intéresser à une série jugée "bas de gamme ", ou que personne n'a vu). Pendant l'« âge d'or » des séries (la décennie 2000), qui a vu la montée des chaînes câblées, et le succès mondial des "séries de qualité ", les critères esthétiques et commerciaux semblaient se rapprocher. Pour l'universitaire, la série HBO devenait synonyme de la série tout court, les séries moins nobles diffusées sur les chaînes commerciales, consignées au passé du médium, étant relativement peu traitées.

Cela n'est pas le seul écueil d'un traitement monographique ou thématique. La série est une forme marchande. Tout commentaire, profane, journalistique ou universitaire, sur un produit culturel identifiable, que ce soit sur ses qualités divertissantes ou sur ses qualités culturelles, contribue à accroître sa valeur d'usage, et à ce titre risque d'intégrer un discours promotionnel, ne serait-ce que de niche. Ce n'est pas pour rien que les premiers commentaires savants sur les séries télévisées comme " huitième art » allaient main dans la main avec la notion publicitaire de "série culte ", les deux correspondant à l'émergence d'un nouveau marché pour des séries classiques disparues de l'antenne, d'abord sous forme de cassette VHS, ensuite de DVD, et enfin de vidéo à la demande ${ }^{16}$. Relayant par la suite les discours journalistiques et éditoriaux, les premières analyses universitaires ont apporté à leur manière une nouvelle légitimité à une forme culturelle jusqu'alors relativement minorée, même méprisée. Avant d'être banalisée, et rendue inopérante, la notion de " série culte " permettait d'insister sur quelques séries classiques qui se détachaient des autres, recyclant le discours de la cinéphilie d'antan. Mais la sériephilie, mot calque, exprime plutôt l'engouement médiatique qui accompagne les pratiques actuelles de consommation - les séries américaines prédominent internationalement en prime time - qu'un prolongement du mouvement cinéphile historique ${ }^{17}$.

\section{Récurrence et pseudo-réalisme}

Revenons au court texte d'Adorno. Ce qui apparait comme une faiblesse en 1954 devrait désormais nous aider à relancer l'analyse des séries sur d'autres bases. La démarche d'Adorno, d'aborder le médium entier à partir d'exemples plutôt anonymes, va radicalement à l'encontre de l'interprétation universitaire de séries (voire d'épisodes de séries) spécifiques, en tant qu'œuvres détachées ${ }^{18}$. Bien que son objet annoncé soit la télévision comme médium, Adorno parle plutôt de quelques fictions qui y ont été diffusées; cela devient même explicit dans la version allemand (Adorno, 2003b : 74). Recadrons donc cet objet: plus exactement, il s'agit de fictions télévisées endogènes en général (les futures séries télévisées), quel que soit le genre. Autrement dit, il faut trouver un niveau d'analyse qui s'applique à la plupart des séries (mais en excluant par commodité les sitcoms et les feuilletons sentimentaux) d'une époque historique donnée ${ }^{19}$. Il est important de préciser que ce niveau d'analyse ne peut être réfuté par quelques contre-exemples empiriques. 
19 Sans le savoir, Adorno met le doigt sur la propriété formelle essentielle de la fiction télévisuelle émergente, à savoir la récurrence et les implications de celle-ci pour le réalisme :

«Ce qui importe, ce n'est pas le crime comme expression symbolique de pulsions sexuelles ou agressives, par ailleurs contrôlées, mais c'est la confusion de ce symbolisme avec un réalisme pédant, dans tout ce qui touche à la perception sensorielle directe. [...] En fait, le pseudo-réalisme permet l'identification directe et extrêmement primitive, telle qu'elle est atteinte par la culture populaire; et il donne l'image d'immeubles banals, de chambres, de robes, de visages, comme s'ils étaient la promesse de quelque chose de palpitant et d'excitant, survenant à chaque instant. [...] La standardisation même, imposée par des cadres de références établis, produit automatiquement nombre de stéréotypes. De même, les techniques de production rendent presque inévitable une certaine stéréotypie. Le peu de temps imparti à l'élaboration des scénarios et l'abondant matériel à produire continuellement induisent certaines formules » (Adorno, $1990: 236$ ).

Même avant la seconde moitié des années 1950, la télévision américaine a quelque chose de structurellement sériel avec les mêmes "cadres de référence", les mêmes personnages stéréotypés. Vu rétrospectivement, la notion adornienne de stéréotype anticipe le personnage récurrent, point d'ancrage de la série classique, qui sert à fabriquer de nouveaux stéréotypes qui se démarquent subtilement des autres. La stéréotypie entre forcément en tension avec les critères de réalisme en vigueur dans les techniques industrielles de production, contraintes par le "peu de temps imparti» imposé par la production très industrielle, et par la brièveté des formats qui empêche « le développement de l'action et des caractères» (Adorno, 2003b: 74) ${ }^{20}$. Le "pseudoréalisme » dont il parle est cependant moins l'apanage de décors pauvres (problème bien réel au début de la télévision ${ }^{21}$ ), que celui d'un irréalisme psychologique résultant de la récurrence de lieux et de personnages (un même type d'énigme criminel affronté par le même résultat, d'où stéréotypie psychologique et une certaine prévisibilité quant au déroulement de l'intrigue). Adorno, lui, parle des indications « au moyen d'un feu rouge et d'un feu vert » (1990 : 237). La série est formellement condamnée à un irréalisme tant psychologique que social, qui s'accentue au fur et à mesure qu'elle s'éloigne de sa forme primitive, le feuilleton domestique. Ce " pseudo-réalisme » n'empêche pas le succès d'une série, au contraire, mais il indique les tensions idéologiques présentes dans ses prémisses ${ }^{22}$. La notion de stéréotype implique une certaine rigidité dans le design des personnages ${ }^{23}$ , une adaptabilité limitée à une stratégie spécifique, elle-même limitée à une certaine conjoncture. Autrement dit, le stéréotype appelle une analyse idéologique et historique, et non morale ou esthétique.

21 L'autre aspect du pseudo-réalisme pointé par Adorno, c'est l'importance jouée par l'assemblage infrastructurel dans le déclenchement des intrigues ${ }^{24}$. Pour cela, des espaces banals doivent forcément promettre quelque chose de "palpitant »: le saloon se transforme " naturellement » en lieu de bagarre; le bureau du chef en lieu de briefing et de transmission; le laboratoire et l'ordinateur de bureau en lieu d'exposition et d'interrogation; la salle banalisée d'interrogation en lieu de confession; l'open space en lieu de rivalités interpersonnelles. De même pour les vêtements, et les manières de se tenir, autant d'indices donnés sur la valeur des personnages avant même qu'un mot soit prononcé. Les décors sont donc des constructions idéologiques, au même titre que les personnages stéréotypés, et participent aux possibilités de la récurrence ; c'est l'ancrage du dispositif (personnages et décors) qui permet celle-ci. Dans les séries westerns par exemple, il s'agit de quelques protagonistes normatifs et quelques lieux «normalement » 
harmonieux, dérangés par des individus «pulsionnels» qui mettent à l'épreuve le dispositif. Voilà formellement exprimé un certain conformisme de comportements et de sens moral en amont de chaque intrigue particulière, ce qui permet la clôture de l'intrigue, la restauration du statu quo ante afin qu'une série puisse se reproduire comme si de rien n'était.

\section{Le concept d'industrie culturelle}

Il est clair néanmoins que, si on veut prolonger l'analyse adornienne de la fiction télévisée, il faudra le faire dans l'esprit, et non à la lettre, surtout en ce qui concerne le concept clé d'industrie culturelle. À cet égard, je citerai Olivier Voirol, propos que je fais mien :

«Tout d'abord, le concept d'industrie culturelle a un caractère historique [...] Il renvoie donc à cette période particulière, celle des années 1930 et 1940 qui, sur le plan de la culture, a vu le développement de l'industrie du disque, du cinéma comme mode de divertissement de masse, de la télévision, des grandes entreprises des médias et de la communication. [...] Si, ensuite, le concept d'industrie culturelle garde son actualité, c'est moins dans son diagnostic que dans l'articulation théorique qu'il suppose et à laquelle il invite. Se référer à un concept revient à endosser les multiples filaments de cette approche, sans quoi cela reviendrait véritablement à "déglinguer" le concept. En restant fidèle à ce sens du concept d'industrie culturelle surgit une approche de la culture dont l'exigence est de croiser économie politique, psychanalyse et esthétique. [...] Enfin, si l'on accepte cette analyse, un recours judicieux au concept d'industrie culturelle doit reprendre ses principales dimensions et donc inscrire une analyse de la culture contemporaine dans une théorie générale des transformations du capitalisme et des formes d'individuation. Le caractère exigeant d'une telle tâche rend donc son actualisation compliquée, car la coopération rendue possible dans le cadre exceptionnel d'un institut interdisciplinaire de recherche fait aujourd'hui défaut. Ceci explique notamment que la plupart des chercheurs contemporains s'inspirant de ce concept tendent à privilégier un aspect au détriment des autres, ou qu'ils s'en tiennent à des interprétations sans chercher à en actualiser leur portée. » (Voirol, 2014: 154-155).

En acceptant seul (à titre provisoire) pour des séries ce défi à triples volets (économie politique, esthétique, psychanalyse), je ferais de mon mieux de ne pas trop « déglinguer » le concept d'industrie culturelle! Je préfère recadrer la dimension esthétique (en raison $\mathrm{du}$ caractère très industriel des séries) en analyse de la forme. Quant au volet de la psychanalyse, je partirai de quelques thèmes révélés par l'analyse idéologique, et non d'une mobilisation de la clinique qui excède mes compétences.

\section{À partir d'Adorno, de Levine et de Marx}

Trois présuppositions sous-tendent mon analyse « néo-adornienne » des séries. D’abord, et décisivement se trouve l'affirmation philosophique d'Adorno dans sa Théorie esthétique que « la forme... est elle-même un contenu sédimenté » (Adorno, 1996: 196) ${ }^{25}$. Dans la tradition de l'économie politique, les instances économiques et idéologiques (les contenus) sont consubstantielles. Je propose donc d'explorer le lien entre l'économique et l'idéologique dans la série à travers la médiation de la forme. À des fins argumentaires, je maintiens que la forme spécifique adoptée par la série (elle-même une forme) à un 
moment historique donné prime l'analyse du contenu d'une série particulière (et a fortiori d'un épisode particulier).

Deuxièmement, dans un livre important de critique formaliste, Caroline Levine propose le terme emprunté du monde du design d'affordance, qui décrit les qualités transférables d'une matière (coton, verre) et des usages potentiels d'un objet (fourchette, poignet, etc.) (Levine, 2015 : 6-11). On pourrait utilement croiser cette notion avec celle d'Adorno cidessus. Forte de sa lecture de Surveiller et Punir (Michel Foucault), Levine affirme que les formes sont toujours « portables » d'un domaine à l'autre, à l'image de l'emploi du temps monastique transposé dans les écoles, les hôpitaux et les prisons. S'ouvre alors la possibilité théorique que l'organisation du marché des valeurs, par exemple, puisse se transférer dans une forme artistique comme la série. Certains genres sériels sont incompatibles avec certaines intrigues, par exemple, la série d'espionnage avec ses missions régulières à l'étranger peut difficilement intégrer des drames personnels, de la vie de famille, etc.

26 Troisièmement, la distinction faite par Marx entre la subsomption formelle et subsomption réelle au capital dans un chapitre «inédit» du Capital (Marx, 2010). La subsomption formelle s'applique lorsque l'intégration marchande intervient après coup, lors de la vente d'un travail recourant aux méthodes de production artisanales. Ainsi l'écrivain « indépendant » qui propose le produit terminé de son travail à l'éditeur ou au producteur ; à celui-ci la charge de réaliser (ou non) sa valeur. Dans la subsomption réelle, le processus de production est directement tourné vers le marché à l'intérieur de ses méthodes de conception, d'élaboration et d'organisation. La distinction est essentielle à la notion d'une périodisation du capitalisme, et de l'approfondissement de sa logique interne. Ainsi, il faut comprendre la subsomption réelle au capital comme un processus historique dont l'horizon absolu serait probablement inatteignable en termes anthropologiques. Jason Read a soutenu que la subsomption réelle s'étend à la production d'une subjectivité capitaliste, une forme d'adaptation existentielle; autrement dit, ce concept (économique) comporte une dimension proprement idéologique (Read, 2003 : 103-151).

La raison d'être de la sérialité, que ce soit sous la forme de série ou de feuilleton, est historiquement liée aux impératifs commerciaux; non seulement elle permet de stabiliser l'audience et de vendre des espaces publicitaires à l'avance, mais aussi de générer de nouveaux contenus régulièrement grâce à des personnages préétablis, en minimisant le risque d'échec. En ce sens, l'assemblage d'une série (personnages, lieux), immanent, joue le rôle d'une "machine " génératrice de récits; en quelque sorte, on pourrait parler de " capital fixe symbolique ». Dans les années 1950 et 1960, on pouvait se contenter des assemblages minimes (un ou deux personnages récurrents), surtout dans les séries westerns, contes de moralités atemporels, qui se diffusent dans les salons d'une société marquée par la croissance régulière et assurée; le rythme de la sérialité, hebdomadaire ou quotidien, témoigne d'une vie faite d'habitudes stables, elles-mêmes récurrentes et programmées. Adorno aurait dit que la soumission à la sérialité programmée est en elle-même une forme de conformisme social.

Dans un premier temps, les chaînes louaient des créneaux horaires aux sponsors uniques, qui sous-traitaient la production à des boîtes indépendantes (à ce titre, Adorno ne mentionne pas que The Four Seasons Playhouse fut sponsorisé par les machines à coudre Singer, avec un bref film publicitaire inséré chaque fois avant l'émission). La relocalisation de la production télévisuelle à Hollywood à partir de 1955 (qui marqua la 
fin du boycott par les studios) a vu la généralisation de la série au format de 52 minutes (qui remplaçait celui de 26 minutes), 8 minutes étant réservées pour des spots publicitaires groupés qui s'intercalaient dans l'émission à des intervalles réguliers ${ }^{26}$. Auparavant, la plupart d'émissions de fiction étaient des captations diffusées en direct, d'où la prédominance du modèle théâtrale (avec son unité du temps et de l'espace) jusqu'aux années 1960 .

Le passage au format de 52 minutes exigeait une intrigue secondaire de rallonge, le plus souvent sous la forme d'un « traitement » interne. L'assemblage étant stable d'un point de vue idéologique, la tension dramatique provient toujours des "visiteurs", de mauvais éléments. Cela implique une conception de la vie en société qui doit beaucoup à l'influence du puritanisme : le terreau du désordre, c'est l'incapacité de certains individus à se maîtriser, et non les conflits d'intérêts réels entre catégories sociales ${ }^{27}$. D'une certaine manière, il y a contradiction entre la série western, qui réaffirme des valeurs traditionnelles face à la peur d'un relâchement moral général que suscite la venue d'une consommation de masse, et les spots publicitaires qui justement poussaient à la consommation sans discrimination, et sans limites.

\section{Les segments modulaires}

Dans les années 1960 s'est produite une évolution formelle dans la série classique, en grande partie pour mieux accommoder les interruptions publicitaires : le découpage de l'intrigue de chaque épisode en segments modulaires pouvant intégrer des ruptures de ton, et détachables de l'intrigue. Un mémorandum pour scénaristes de la quatrième (et dernière) saison de la série d'espionnage Agents très spéciaux (1964-68) a précisé : «Il faut bien comprendre qu'à aucun moment l'humour ne devrait pas gêner l'intrigue ou annuler le suspense... [Les intrigues] doivent être crédibles, conséquentes, glamoureuses, sophistiquées, avec un rythme rapide, pleines de surprises et entrelacées d'humour... [Les protagonistes] ne devraient jamais plaisanter alors qu'ils sont en danger. » (Jon Heitland, 1987 : 188-189). Une autre évolution formelle corollaire, ce sont des personnages sans profondeur psychologique, des «machines idéologiques » : autant de stéréotypes comme l'espion mondain et désinvolte, le savant fou, le nouveau riche vulgaire et mégalomane, le vieux bourgeois réactionnaire, la femme professionnelle « coincée » ou la paysanne naïve qui ne demande que d'être dégourdie... Cette évolution fut essentielle pour la conquête du marché international (dans un premier temps, occidental), qui commençait à s'ouvrir : l'absence relative de dimension psychologique dans les séries d'espionnage a fait en sorte qu'elles étaient mieux à même de pénétrer des marchés étrangers. En d'autres termes, des personnages moins enracinés dans la culture américaine (comme dans le western), qui faisaient « modernes $»^{28}$.

31 L'organisation en segments modulaires permet en premier lieu la séparation entre le " siège ", où les personnages récurrents existent pour eux-mêmes, et le monde extérieur où ils peuvent démontrer les compétences (agilité physique et intellectuelle, sophistication) affirmées sans opposition au siège, véritable point d'ancrage idéologique. Cette structure formelle implique que les personnages récurrents soient porteurs d'un projet social positif, qui donne du sens à leurs agissements dans le monde, en commençant par la légitimation du recours à la violence et au subterfuge. 


\section{La série feuilletonnante : synthèse de la série et le feuilleton}

Les segments modulaires ont assumé une autre fonction dans les années 1980. La série Hill Street Blues (1981-7) marque l'émergence de la série feuilletonnante, synthèse instable entre la série à épisodes autonomes et le feuilleton ${ }^{29}$. Prolongé par NYPD Blue (1993-2005), ce type de série hybride intègre avec des valences diverses la série, le feuilleton, la sitcom et le soap opera. Une de ses caractéristiques est l'inflation des personnages récurrents (44 dans Hill Street Blues, dont 20 principaux).

Dans X-Files (1993-2002), on trouve des épisodes autonomes à la manière de la série classique, et des épisodes espacés qui reprennent la grande mythologie de la série, à savoir une conspiration liant les autorités et des extraterrestres. X-Files a été l'une des premières séries à expérimenter l'arc narratif, où les éléments d'une grande narration statique reviennent au cours de la saison. Depuis, de nombreuses séries ont davantage mis en feuilleton l'arc narratif avec des bouts d'intrigue qui se reprennent de manière espacée (Mentalist, 2008-15) ; cela est même devenu le format standard. Mais c'est dans 24 heures chrono (2001-10) que la feuilletonnisation est poussée jusqu'au bout : soit une saison de 24 épisodes où chaque épisode représente une heure en temps réel (moins les pauses publicitaires). Une variante récente est la série feuilletonnante/anthologique où les personnages et les lieux récurrents changent d'une saison à l'autre, où seule subsiste la « marque » (Fargo, True Détective).

Alors que la série classique à épisodes autonomes est idéalement adaptée au héros masculin (seul ou avec partenaires) intervenant dans la vie des autres et démontrant sa maîtrise de la situation, la série feuilletonnante ne peut fonctionner sans que celui-ci n'expose à son tour sa vie privée qui, pour être crédible sur le plan dramatique, doit dévoiler ses faiblesses, blessures, doutes. Les innovations formelles (split-screen, horloge numérique) de 24 heures chrono servent par-dessus tout à « remasculiniser » le feuilleton ouvert (Tara McPherson, $2007: 174)^{30}$, forme qui oblige le héros Jack Bauer à courir après son affaire dans un état d'impuissance relative ; il s'en sort (et le monde avec) chaque fois in extremis et avec beaucoup de réussite. Autrement dit, si on enlève la stricte continuité temporelle (expérience extrême), rien (ou pas grande chose) ne distingue la série feuilletonnante du soap sur le plan formel, si ce n'est pas la difficulté de se reproduire audelà de cinq ou six saisons.

Déjà dans les années 1980, Miami Vice avait mis à mal la série classique avec la passivité de son propos; l'impossibilité de la lutte contre le vice (drogue, sexe, finance) amène les deux flics principaux à prendre la pose romantique sur fond de ralentis esthétisants. Le souci du réalisme n'opère plus : comment jouer le rôle de faux trafiquant dans le monde de la pègre, semaine après semaine, en restant crédible ? Comment incarner un projet positif quand on sait que l'économie du pays est dépendante de l'argent provenant du trafic de la drogue ? C'est donc le feuilleton « raisonné » (encadré par la notion de saison), expérimenté par les chaînes de câble dans les années 2000, qui s'avère être la forme la mieux adaptée pour exprimer cette passivité essentielle, où les protagonistes sont emportés par une situation qu'ils ne maîtrisent pas. L'organisation en saisons, qui permet des résolutions partielles, ou des changements de direction, sert surtout à maintenir la tension dans le feuilleton ouvert, traditionnellement de basse intensité ${ }^{11}$. Une autre innovation de 24 heures chrono, c'est l'extrême indétermination des personnages ; ceux-ci 
peuvent trahir ou subir une mort violente à n'importe quel moment, d'où l'importance structurelle du grand réservoir de personnages dans Game of Thrones (257 nommés dans la saison 3).

Que signifie le passage à la série feuilletonnante ? À tout le moins, une évolution dans le contexte social (y compris les modes de réception) qui a assuré le succès de la série classique. De toute évidence, l'assemblage simple à même d'accueillir des intrigues à la fois variées et finalisées n'opère plus à partir des années 1990, qui voient la consécration des séries "décentrées ». Première remarque : les personnages multiples, représentant des opinions et des approches très diverses des problèmes sociaux, permettent de limiter les dégâts d'une passivité structurelle, d'une absence de projet social à défendre, et pendant d'une même passivité face au monde de la finance. Deuxième remarque : en eux-mêmes, les personnages multiples déplacent les sous-intrigues vers les relations endogames au détriment du « social ", ouvrant de fait la forme série vers celle du feuilleton. Troisième remarque: obligée de faire évoluer les personnages d'un épisode à l'autre, la forme feuilletonnante introduit des éléments d'instabilité dans une série, qui est condamnée à un combat anti-entropique dès le départ. L'assemblage ne peut que se dégrader; c'est une forme qui correspond à un monde vu en ces termes.

\section{La subsomption réelle de la forme série}

La théorisation de cette évolution en termes de subsomption réelle peut maintenant se faire sur plusieurs axes. D'abord, le département de marketing s'implique désormais dès le stade de la conception. Comme le décrit le sociologue John Caldwell, après une étude de terrain :

« N'importe quel scénario ou projet élaboré pour la télévision aux heures de grande écoute ou pour le cinéma grand public de nos jours mobilise fortement, dès les premières réunions de scénaristes et de producteurs, des managers provenant des départements de gestion, de marketing, de contrats internationaux, de distribution, de commercialisation et de nouveaux médias. [...] Lors des étapes de proposition et d'écriture, les idées de scénario seront développées comme des divertissements aux supports variés. » (Caldwell, $2008:$ 232-233).

Autrement dit, le consommateur est déjà conceptualisé et ciblé comme une partie intégrante du processus de production. Dans le cas de Lost (2004-10), le producteur ABC a collaboré avec des sites amateurs dédiés à la série afin d'établir une base de données des fans, mobilisables comme des auxiliaires de marketing bénévoles. Sur les réseaux sociaux, les séries fournissent du lubrifiant culturel pour l'établissement des profils nécessaires à la conversion des usagers en fournisseurs de données. Perçue ainsi, la série devient une forme de capital marque. Parlant de plusieurs séries HBO, Michael Szalay affirme que "des drames sont des marchés à terme [...] et devraient se comprendre comme des équivalents fonctionnels de la catégorie d'outils financiers appelés dérivés » (Szalay, $2014: 112)$.

Deuxièmement, la subsomption réelle s'exprime dans la façon dont la production capitaliste se reflète de plus en plus dans la forme série. Dans la série classique, il s'agit de réinvestir l'assemblage de manière cumulative, semaine après semaine (croissance incrémentielle). Mais en tant que processus continu de création de survaleur, le capitalisme dépend de la croissance composée, d'où le besoin de se réinvestir de manière exponentielle. Le capital fictif (échange de valeurs n'existant que sur le papier) a toujours 
fait partie de l'économie capitaliste, mais jamais au point actuel ; il s'agit d'une évolution structurelle de celle-ci dans un stade financier ou "tardif ", où le maintien du système l'oblige à créer sans cesse de nouvelles valeurs fictives pour éponger la survaleur. C'est là que se trouve le pendant formel de la série qui multiplie des personnages et des situations pour en arriver à Game of Thrones (2011-), avec ses certaines de personnages virtuellement récurrents, et virtuellement liquidables à tout instant, et dont la valeur ne sera jamais réalisée. Chaque série est désormais un marché de valeurs spéculatives, où se gèrent en interne des "placements» de personnages et de sous-intrigues, dont la rentabilité s'évalue en cours de route. À la différence des personnages secondaires dans la série classique, «loués» et "rendus» à la fin de l'épisode, ceux créés dans la série feuilletonnante ne disparaissent pas s'ils ne sont pas mis à mort, parfois en masse (comme dans une correction boursière).

Troisièmement, la série feuilletonnante tend à imiter le capitalisme financier dans sa structure interne. De multiples trames d'intrigues, qui exigeront un rythme constant d'accumulation, sont lancées dès le premier épisode (huit dans le premier épisode de la troisième saison de 24 heures chrono). La concaténation des intrigues ressemble formellement à l'imbrication des circuits de capital-argent et capital-marchandise discutés au début du Capital, volume 2. Le recours au crédit sous la forme de nouveaux personnages et sous-intrigues est essentiel pour lisser la circulation des éléments narratifs, et pour éviter des ralentissements de rythme, voire des ruptures paralysantes.

41 En termes "d'affordance » (Levine, 2015), le format qui correspond le mieux à la série feuilletonnante est la saga, modernisée (Les Sopranos) ou non (Game of Thrones). L'inflation des personnages est amortie par la mise en réseau, que ce soit la famille, le clan, le gang, l'organisation, l'institution, le cabinet d'avocats. Loin d'être des agents libres, les personnages créés sont contenus par des sous-intrigues, autant de chaînes de production fictive. Sans cela, une série comme Game of Thrones s'écroulerait sous son propre poids ; en tout état de cause, la série feuilletonnante peine à se reproduire au-delà de six ou sept saisons. 24 heures chrono en est un bon exemple avec sa logique de surenchère; la première saison a notoirement épuisé sa trame narrative initiale après huit épisodes, faute de sous-intrigues suffisantes. Au fond, le problème formel de maintien du rythme est la conséquence de la passivité idéologique décrite ci-dessus, qui condamne les protagonistes récurrents à réagir sans cesse dans un monde où «personne n'est innocent $»^{32}$.

\section{Psychanalyse. Sadomasochisme...}

« [La tendance à canaliser la réaction de l'audience] converge avec un soupçon largement partagé [...] selon lequel la majorité des émissions télévisées visent aujourd'hui à produire, ou au moins de reproduire, la suffisance, la passivité intellectuelle et la crédulité qui semblent s'accorder avec des crédos totalitaires, même si le message explicite des spectacles est antitotalitaire. [...] [Cette "psychanalyse à l'envers" (Lowenthal)] implique en quelque sorte que le concept psychanalytique d'une personnalité complexe a été récupéré par l'industrie culturelle, et que cette conception est utilisée dans le but de piéger le consommateur et de l'engager de manière psychodynamique au service d'effets prémédités. » (Adorno, 1990 : 232).

Exposée ainsi, sommairement, la notion de psychanalyse appliquée qu'avance Adorno ici reste programmatique, et très générale, trop sans doute pour en tirer une méthode. Mais 
elle contient deux pistes intéressantes: le décalage, voire la contradiction entre le message explicite, généralement consensuel, et le message "caché ", inconscient, plus trouble; "la psychanalyse à l'envers", vulgarisée, qui permet de dessiner des personnages non lisses, complexes, prêts-à-agir, qui échappent néanmoins aux intentions des créateurs. En voilà l'esquisse d'une synthèse des deux modalités de l'inconscient: freudienne et idéologique. Je reprends ici mon commentaire supra sur l'exemple fourni par Adorno: l'ex-gangster et propriétaire d'une boîte abritant un casino illégal Willie Dante, séducteur, débonnaire, mais viril, même brutal quand il le faut, personnage non lisse qui fait la synthèse des qualités masculines traditionnelles et de l'ouverture envers le monde du plaisir et de la consommation (même s'il pousse ce dernier trait un peu au-delà des limites légales). Sur ce plan, Dante rappelle l'homme viril pointé déjà par Adorno dans Minima Moralia :

«Il y a lieu de se défier de certaines formes de certaines poses affichant la virilité. [...] Il semble que cette violence vise les autres..., mais il s'agit en vérité d'une violence que [ce genre de héros] s'est faite à lui-même. [...] Ces mâles seraient donc bien, au fond d'eux-mêmes, ce qu'en fondent le plus souvent les scénarios de films qui les mettent en scène, des masochistes. Leur sadisme est mensonge, et ce n'est qu'autant qu'ils mentent qu'ils deviennent véritablement des sadiques, c'est-à-dire des agents de la répression. » (Adorno, $2001:$ :46-47).

Les tough guys ont un besoin impératif de transformer d'autres sujets en objets, pour ne pas reconnaître qu'ils leur ressemblent. Comme le dit un terroriste islamique à Jack Bauer dans 24 heures chrono: "Personne n'est innocent. Je crois en ce que je crois aussi fortement que vous croyez en ce que vous croyez ${ }^{33}$. Logiquement, ce genre d'affirmation, impossible à contrer, neutralise toute dimension politique, celle-ci étant réduite aux jeux d'intérêt et aux rapports de force, soit un gain ou une perte, selon le point de vue. Dans un monde incontrôlable, on ne peut que réagir au désir de l'autre. Selon les mots larmoyants de Jack Bauer, « vous savez ce que je regrette le plus ? C'est que ce monde ait besoin de quelqu'un comme moi. ${ }^{34}$ » Transformer les autres en objets implique qu'on se transforme également en objet à leurs yeux. À ce titre, 24 heures chrono, série exagérée jusqu'à l'absurde, permet de rendre lisible des symptômes influant sur la normalité, elle-même pathologique. Que ce soit dans le western, la série d'espionnage et la série policière, les héros récurrents imposent leur violence aux autres avec une force légitime, mais au cours de l'intrigue ils subissent à des degrés divers la violence des autres (classiquement, dans la série d'espionnage, le héros se fait torturer avant de retourner le sadisme de l'autre contre lui-même). Comment ne pas penser ici au modèle du fantasme sadomasochiste élaboré par Freud, «un enfant est battu», structuration primitive qui vaut pour tous les fantasmes? La jouissance sadique provoquée par l'idée d'un homme (ou plus rarement d'une femme) allongé et écartelé, métaphoriquement ou non, est sauvée d'un basculement dans le déplaisir culpabilisant par le versant masochiste du fantasme, où on se met à la place de la victime (Freud, 1973 : 219-243).

Le sadisme et le masochisme, qui s'alignent sur le couple actif/passif, sont donc inséparables, reliés dans une dynamique dont la valence est plus forte tantôt d'un côté, tantôt de l'autre. Dans la série classique, le versant masochiste est minoré, la violence légitime est toujours triomphante à la fin, et avec elle, les valeurs de l'idéologie dominante. Dans un mémorandum aux scénaristes de la série western Bonanza, on explique que «les Cartwright ne sont pas de bonnes âmes. Un problème ne doit jamais devenir le leur simplement parce qu'ils ont fourré leur nez dedans. » Autrement dit, la menace contre l'ordre est toujours d'abord et avant tout une menace personnelle contre 
les Cartwright, personnages récurrents, qui attisent et subissent celle-ci, sans dévoiler des faiblesses.

C'est justement la passivité structurelle si caractéristique de la série feuilletonnante qui nous plonge dans un autre monde, où les tough guys subissent les affres d'une vie privée qu'ils ne maitrisent pas, pas plus que leur rôle social, marqué sur le fond par l'échec. C'est le versant masochiste qui domine donc in fine, malgré la violence expéditive employée contre des terroristes, ou des criminels de plus en plus pervers, irrationnels. La passivité structurelle provient d'une situation dans laquelle l'alliance traditionnelle entre les valeurs puritaines et les valeurs de la libre entreprise s'est défaite; comme le dit avec autorité et cynisme un financier dans un épisode de Miami Vice $e^{35}$, tout le système bancaire est dépendant du commerce de la drogue. Un abordage sociologique (et non fataliste) dans les séries, qui supposerait la mise en place parallèle de politiques sociales dédiées contre l'action aveugle du marché, semble impensable. Un policier peut éliminer un trafiquant par-ci, par-là, sans prétendre combattre un mal systémique, qui bénéficie de la complaisance, voire de la complicité des concitoyens qu'il est censé défendre.

Dans X-Files, les extraterrestres, avec la collaboration du pouvoir, projettent le remplacement en 2012 de l'espèce humaine par une nouvelle espèce métissée avec l'ADN extraterrestre. Les protagonistes Fox Mulder et Dana Scully mènent alors une enquête vaine, condamnée d'avance, et dénuée de tension sexuelle entre les deux "partenaires ». Dans un monologue étonnant, Scully demande : «Notre espèce est-elle condamnée, elle aussi, à disparaître dans la sixième extinction que nous annoncent déjà les scientifiques ? [...] Tout cela finira un jour, la vie n'engendrant plus la vie ? La Terre devenant stérile comme les étoiles, comme le cosmos ? ${ }^{36}$ " En attendant la fin, les deux protagonistes assistent, impuissants, à l'hystérisation du monde, traduite en autant de symptômes qui défient toute logique, qui provient d'une culpabilité primitive, d'un besoin inconscient de punition. Comme l'a dit Fredric Jameson: «Il nous semble plus facile aujourd'hui d'imaginer la dégradation complète de la Terre et de la nature que la dégradation du capitalisme tardif. » (Jameson, 1994 : xii). Autrement dit, il est plus facile d'imaginer la fin du monde que la fin du capitalisme.

Dans Les Experts et d'autres séries légistes (Bones, NCIS) des années 2000, ce masochisme moral s'incarne dans les scènes d'autopsie, où la permutation entre sujet et objet dans le fantasme sadomasochiste permet la jouissance sadique (et scopique) devant le découpage légiste d'autrui ${ }^{37}$, et le déplaisir déculpabilisant provenant du fait que le cadavre sur la table mortuaire pourrait être le nôtre. La science s'applique non pas à une société en évolution, mais «inutilement ", après coup, à ceux qui sont seuls dignes d'elle, à savoir les morts. D'autres séries encore se situent après l'effondrement de la civilisation, où les rapports sociaux sont réduits à la lutte pour la survie, contre un ennemi zombi qui existe au-delà de l'énonciation, en pure vengeance (The Walking Dead). En deçà de ces cas extrêmes se trouvent maintes séries impliquant des avocats (The Good Wife; Suits) ou des professionnels antisociaux (House), où le jeu social tend à se réduire à la guerre de chacun (e) contre chacun $\left(\mathrm{e}^{38}\right.$.

\section{... et paranoïa}

Un certain niveau de paranoïa est inhérent à la forme série ${ }^{39}$. Confronté semaine après semaine à une menace ou à un danger, le personnage récurrent est naturellement prédisposé à la méfiance envers des étrangers, d'autant que ceux-ci ne sont là que pour 
semer le trouble (et donc pour déclencher l'intrigue). Il n'est pas surprenant qu'Adorno ait été frappé par le personnage sériel embryonnaire de Dante, pour qui le succès (et le maintien) de son casino illégal dépend de sa capacité à renifler des flics, des trouble-fêtes, et des pipelettes. Quant à la série d'espionnage des années 1960, les agents récurrents ont bien raison de se méfier de tout le monde lors d'une mission à l'étranger.

Cette paranoïa « structurelle » se radicalise avec la venue de la série feuilletonnante et de la passivité idéologique qui l'accompagne. On peut mesurer cette radicalisation en comparant deux séries emblématiques: Les Envahisseurs (1967-68) et 24 heures chrono (2001-10). Dans Les Envahisseurs, les extraterrestres sont sur Terre et ont pris forme humaine; seuls quelques menus indices physiques permettent de les identifier (absence de saignement, léger raidissement du petit doigt). Tout le monde est donc logiquement suspect, sauf les gens reliés par des liens de sang ou d'amitié de longue date. Dans un épisode ${ }^{40}$, le protagoniste récurrent David Vincent va jusqu'à écarter d'office le juge des possibles imposteurs extraterrestres; il existe quand même des limites idéologiques à la paranoïa autorisée. Dans 24 heures chrono, l'agence antiterroriste grouille de traîtres; même des amis intimes, et de longue date peuvent passer silencieusement à l'ennemi. Pratiquement tous les collaborateurs de Jack Bauer finissent par trahir, lui et leur pays. Dans la saison 6 (2007), l'ultime digue cède, celle de la famille : ce sont le père et le frère de Jack Bauer qui étaient derrière l'assassinat du président Palmer. Désormais, la paranoïa n'a plus de limites; un autre président des États-Unis, Logan, s'avère être un traitre de mèche avec des terroristes, contre ses concitoyens.

"Ne faites confiance à personne", disait déjà Fox Mulder (The X-Files) dans les années 1990, c'était même son mot de passe («TrustNo1»). Pour l'irascible Docteur House (House ), l'établissement d'un diagnostic ne doit pas tenir compte des dires des malades, trop menteurs. De même pour les légistes des Experts, pour qui seuls les morts disent la vérité ("Les gens mentent. C'est pourquoi on ne peut se fier qu'aux indices et à la science ») Le sermon que fait à ses équipiers Jason Gideon, chef de section de profileurs du FBI ( Esprits criminels) est typique : «On ne connait pas vraiment nos voisins. Il promène son chien. Il jardine le dimanche. C'est quelqu'un qui, en apparence, aime être seul. Mais à y regarder de plus près, on s'aperçoit qu'il tourne toujours autour des gosses du quartier. Il passe inaperçu parce qu'on ne le considère pas comme une menace. [...] Et puis on a su que les inconnus ne représentent qu'une fraction du nombre total des agresseurs [sexuels des enfants]. On croise la plupart d'entre eux tous les jours. C'est votre famille, vos voisins, ou alors des enseignants $\aleph^{42}$. Comme par hasard (mais l'idéologie ignore le hasard), il oublie les curés...

51 La paranoïa devient une réaction normale alors que les relations marchandes ont investi quasiment tous les domaines de la vie sociale, chacun agissant par calcul (en le niant), mû par son propre intérêt, son propre désir et rien d'autre. La double vie avec ses petits secrets, révélée par l'enquête, est la norme. Seuls le policier et ses variantes (légiste, médecin) peuvent tirer de leur expérience des vérités sur la société dans sa totalité, d'autant que leur équipe est un modèle de désintéressement et d'application dans un monde ravagé par l'égoïsme. La même basse contrainte historique se fait sonner : le crime s'explique par le banal appétit de gain, ou mieux, par la pulsion sexuelle perverse, l'un ou l'autre ou les deux ${ }^{43}$. L'idée de société ne survit qu'en tant que lieu de crime géant où tout le monde est suspect; la méfiance, voire l'hostilité envers les autres est la condition même de toute interaction sociale maîtrisée. 

où le monde s'explique par des figures de personnes rapidement esquissées, par la rationalisation pauvre qu'offrent des stéréotypes sans vraie dimension sociale. La société à la dérive mortifère est la projection narcissique d'une catastrophe psychique interne.

\section{BIBLIOGRAPHIE}

Adorno Th. W., 1990, « La télévision et les patterns de la culture de masse », Réseaux, vol. 9, no. 44-45 (publication originale, 1954).

Adorno Th W., 1996, Théorie esthétique, Paris : Klincksiek.

Adorno Th W., 2001, « Tough baby » in Minima Moralia, Paris : Payot (publication originale, 1951).

Adorno Th W., 2003a, « Prologue sur la télévision » in Modèles critiques, Paris : Payot (publication originale, 1953).

Adorno Th W., 2003b, « La télévision comme idéologie » in Modèles critiques, Paris : Payot (publication originale, 1953).

Adorno, Th W., 2003c, « Recherches expérimentales aux États-Unis » in Modèles critiques, Paris : Payot (publication originale, 1968).

Benassi S., 2000, Séries et feuilletons TV. Pour une typologie des fictions télévisuelles, Liège : Éditions du Céfal,

Brooks T., Marsh E., 1995, The Complete Directory to prime-time network and cable TV shows, New York : Ballantine (publication originale, 1979).

Buxton D., 2010, Les Séries télévisées. Forme, idéologie et mode de production, Paris : L'Harmattan.

Caldwell J. T., 2008, Production Culture, Durham NC. : Duke University Press.

De Baecque A., 2003, La Cinéphilie. Invention d'un regard, histoire d'une culture 1944-1968, Paris : Fayard.

Freud S., 1954, « Remarques psychanalytiques sur l'autobiographie d'un cas de paranoïa (le président Schreber) », Cinq psychanalyses, Paris : PUF (publication originale, 1910).

Freud S., 1973, « Un enfant est battu », Névrose, psychose et perversion, Paris : PUF (publication originale, 1919).

Heitland J., 1987, The Man from UNCLE Book, New York : St. Martins Press.

Horkheimer M., Adorno Th W., 1974, La Dialectique de la raison, Paris : Gallimard (publication originale, 1944).

Hullot-Kentor R., 2006, Things beyond ressemblance. Collected Essays on Theodor W. Adorno, New York : Columbia University Press.

Jameson F., 1994, The Seeds of Time, New York : Columbia University Press.

Levine C., 2015, Forms. Whole, Rhythm, Hierarchy, Network, Princeton N.J. : Princeton University Press. 
Macherey P., 1966, Vers une théorie de la production littéraire, Paris : Maspero (d'autres éditions depuis).

Marx K., 2010, Le Chapitre VI. Manuscrits de 1863-1867, Paris : GEME/Éditions sociales (publication française originale, 1971).

Mattelart A., 2015 (2005), Diversité culturelle et mondialisation, Paris : La Découverte.

McPherson T., 2007, « Techno Soap. 24, Masculinity and Hybrid Form », in Steven Peacock (dir.), Reading 24, London : I. B. Taurus.

Morris M., 1988, « Banality in Cultural Studies », Block, nº 14, London : Middlesex Polytechnic. Müller-Doohm S., 2004, Adorno, Paris : Gallimard.

Parks R., 1982, The Western Hero in Film and Television, Ann Arbor, MI : UMI Research Press. Read J., 2003, The Micro-Politics of Capital, Albany N.Y. : State University of New York Press. Szalay M., 2014, « HBO’s Flexible Gold », Representations, nº 126, Berkeley CA. : University of California Press.

Thompson J. O., 1993, « Up Aporia Creek », in Manuel Alvarado, Edward Buscombe, Richard Collins (dirs), The Screen Education Reader : Cinema, Television, Culture, Basingstoke : Macmillan (publication originale, 1979).

Voirol O., 2014, «L'industrie culturelle comme diagnostic historique », Illusio 12/13, Théorie critique de la crise, volume 2, Lormont : Le Bord de l'Eau.

Winckler M., 2002, Les Miroirs de la vie, Paris : Le Passage.

\section{NOTES}

1. Selon la jolie formule d'Alexander Neumann.

2. Dans une lettre à Max Horkheimer du 19 avril 1953, Adorno parle d'un esclandre humiliant entre lui et Hacker lors d'une réunion du personnel. D'après Hacker, Adorno ne s'intéressait qu'à ses propres recherches, ne s'identifiait pas à la clinique, et devrait s'occuper plutôt de ce qui avait un intérêt pour les psychologues thérapeutes de la Fondation, «si stupides que ces travaux puissent être [pour lui]». Voir Stefan Müller-Doohm, 2004 : 567. Cela accrédite l'idée que, dans sa conception même, la recherche d'Adorno fut un compromis entre son approche propre et la politique de la Fondation Hacker.

3. Il n'est pas sans intérêt de se pencher sur les collaborateurs remerciés dans la présentation de l'article américain: George Gerbner (1919-2005), qui deviendra doyen de la célèbre Annenberg School for Communication (Pennsylvanie), sera très connu pour ses recherches sur la manière dont la télévision transforme la perception des catégories sociales, en positif ou en négatif; Bernice T. Eiduson (1923-85), qui publiera notamment sur le monde psychologique des scientifiques; Merril B. Friend (1923-), qui écrira sur l'hypnothérapie dans le traitement d'alcoolisme. L'apport réel de ceux-ci à l'étude est impossible à déterminer, mais leur seule mention indique bien un cadre académique et psychologique.

4. En guise de conclusion, Adorno endosse le projet libéral de «transformer ce médium avec des potentialités de grande envergure » (Adorno, $1990: 240$ ). Dans la version allemande du texte, il va jusqu'à flotter l'idée d'une "commission de sociologues, de psychologues et d'éducateurs, responsables et indépendants » qui pourraient indiquer « les provocations et les stéréotypes [...] susceptibles d'abêtir, d'estropier psychologiquement et d'abrutir idéologiquement le public » (Adorno, 2003b : 86). 
5. Les contenus de La Dialectique de la Raison, écrits entre 1941-44, ont d'abord circulé sous forme miméographiée, en langue allemande. Pour la publication américaine en 1947, le moindre terme à consonance marxisante a été systématiquement euphémisé par Adorno et Horkheimer euxmêmes. Voir Robert Hullot-Kentor, 2006 : 24-25. Notons que le terme «idéologie » n'apparaît qu'une seule fois dans une note de l'article de 1954, dans un sens non politique («l'évolution de l'idéologie de l'extraversion ", Adorno, 1990 : 241).

6. Écrit dans la foulée, et publié en allemand en 1953, ce texte recoupe largement l'article " américain ", mais il est sensiblement plus court, et en plusieurs endroits, remanié. Grosso modo, c'est la première partie de l'article américain qui manque (p. 227-33), et avec elle, toute la problématique des effets.

7. Notons le titre américain, passablement normatif : « How to Look at Television ».

8. Stéphane Benassi distingue entre l'anthologie et la collection, la première étant « encadré(e) par une séquence introductive et une séquence conclusive extradiégétique de présentation monologale ", comme dans Alfred Hitchcock Presents (Stéphane Benassi, 2000 : 136). Je préfère garder le terme anthologie pour ce qui était plutôt deux variantes de la même forme, d'autant que l'anthologie Four Seasons Playhouse représente à mon avis une troisième variante, le cycle de comédiens récurrents.

9. Charles Boyer, David Niven, Ida Lupino et Dick Powell (qui joue Willie Dante entre autres).

10. On a diffusé pas moins de 120 séries westerns aux États-Unis pendant les années 1950 et 1960. Voir Rita Parks, 1982.

11. https://www.youtube.com/watch?v=ca6Nsmak9fs\&t=1286s

12. Dans la version allemande de son étude, Adorno précise qu'il a travaillé à partir des scénarios dactylographiés (2003b : 73), obtenus pour lui par Hacker (2003c : 296), mais le compte-rendu ici donne la forte impression qu'il a effectivement visionné l'épisode en question («quand nous voyons »). S'agit-il-là d'une exception? On ne saura jamais. Reste la possibilité que l'épisode diffusé diffère du scénario écrit, mais est-ce que celui-ci aurait stipulé que la femme soit " outrageusement maquillée »? En tout cas, elle ne l'est pas, sauf dans le regard d'Adorno! La décision de travailler sur des scénarios et non sur les programmes diffusés, à moins qu'Adorno n'eût pas accès à un téléviseur, parait bizarre ; à tout le moins, c'est la spécificité de la télévision comme médium qui passe à la trappe. Adorno s'en explique en termes de confort d'analyse, sans vraiment convaincre ; il avait peut-être (et tout simplement) du mal à suivre des émissions en américain en direct. L'analyse à partir des scénarios peut expliquer pourquoi il a parlé de fictions de quinze minutes, format qui n'a jamais existé (Adorno, 1990 : 237).

13. Le code d'honneur en vigueur dans la Frontière (au-delà de la loi d'État), à savoir le gunlaw, permettait de dégainer son revolver, à condition que l'adversaire soit également armé et de face. Dans les fictions télévisées, on dirait que ce code était toujours en vigueur à Los Angeles dans les années 1950, comme dans un western. Le policier sériel embryonnaire avait manifestement besoin de résolutions de ce type, qui permettait au protagoniste de s'en sortir d'une situation compromettante, sans passer par les contraintes légales qui auraient plombé la narration.

14. Être client régulier d'une boîte de jazz, un verre à la main, n'empêche aucunement Gunn de mener ses enquêtes avec son ami, lieutenant dans la police. Ainsi la série réussit à marier les valeurs hédonistes de la consommation avec les valeurs légitimistes et sécuritaires, topos radicalisés dans certaines séries d'espionnage des années 1960. Dans les années 1950, cette synthèse contradictoire est structurelle, surtout dans les séries westerns, dont la culture puritaine et moralisatrice est encadrée par les annonces publicitaires incitant à la consommation sans freins. Plus généralement, dans Buxton (2010), j'insiste sur l'ambiguïté idéologique objective de toute série.

15. Cette critique s'étend à la notion de récepteur « actif », voire « résistant », très influente dans les cultural studies dans les années 1980 et 1990. Armand Mattelart écrit: "L'héroïsation néopopuliste du récepteur résistant a rejoint l'apologie néolibérale du consommateur atomisé » 
(Mattelart, 2015 : 74). L'effet est de neutraliser la charge idéologique, voire d'assumer un artefact commercial américain comme un "opérateur d'universalisation ", où chaque culture peut se redéfinir librement (Mattelart, 2015 : 75). Comme le dit la critique australienne Meaghan Morris : « Dès lors que « le peuple » est en même temps l'autorité interprétative d'un texte, et la figure de l'activité critique, l'approche populiste est au fond non seulement circulaire, mais [...] narcissique » (Morris, $1988: 20$ ).

16. Le travail de légitimation des séries anglo-saxonnes était initié en France en 1989 par les éditions Huitième Art (filiale des Nouvelles Éditions Oswald (NéO), spécialisées dans la littérature fantastique, policière et de science-fiction). Dans un premier temps, elles ont privilégié de manière cinéphile un corpus de séries déjà classiques et plutôt britanniques, dont Le Prisonnier (1967-8), présenté comme un chef-d'œuvre «télévisionnaire» s'inscrivant dans la lignée du surréalisme. Le directeur des NéO, Pierre-Jean Oswald (1931-2000) avait précédemment édité de la poésie surréaliste et des pièces de théâtre militantes. Dans une deuxième vague, le romancier à succès Martin Winckler (2002), très présent dans les médias, y a apporté un mélange d'érudition et d'enthousiasme péremptoire, en insistant de manière discutable sur les qualités progressistes (au sens « libéral ») des séries américaines en général. La critique universitaire était rare avant les années 2000, avant de devenir rapidement pléthorique. Il n'est pas certain que celle-ci, globalement dans la tradition des cultural studies, représente une avancée substantielle par rapport à l'intervention de Winckler, qui a réussi à imposer sa façon d'intégrer les séries dans un discours plutôt philo-américain, où la tradition critique, trop « négative », n'a pas été bienvenue. 17. Les raisons pour cela méritent analyse et débat. Le mouvement cinéphile a trouvé une traduction matérielle sous la forme de ciné-clubs, de revues, et ensuite de rubriques médiatiques, de collections, d'espaces dédiés à la télévision, et décisivement dans la mise en place de cursus de cinéma dans les universités (Antoine de Baecque, 2003). Dans les années 1950, la cinéphilie a canalisé à sa manière l'engagement militant, d'où des ciné-clubs étiquetés communistes, progressistes, catholiques et de droite, et des débats vifs attenants. Rien de tel dans le cas des séries ! De façon plus générale, par sa capacité à introduire de nouveaux critères discriminants, la cinéphilie a exprimé les prétentions culturelles d'une nouvelle classe moyenne (enseignants, cadres moyens, journalistes, etc.) dans une forme relativement méprisée, devenue du coup intellectualisée et socialement porteuse (à l'instar du jazz, du roman policier et plus tard, le rock). Par contre, la tentative de créer des revues spécialisées « sériephiles » a été un échec, ainsi que des tentatives de s'organiser en associations. Plusieurs explications, qui ne sont pas mutuellement exclusives, se proposent dans le désordre : la non-existence d'une culture d'auteurs; la grande disponibilité en rediffusions et en DVD sous licence commerciale ; des productions esthétiquement standardisées; l'absence de dimension politique, mobilisatrice; le démarquage des pratiques de consommation d'une culture de classe (Bernard Lahire).

18. Adorno fournit la justification suivante de cette approche : «On peut également justifier la méthode qualitative par le fait que les produits de l'industrie culturelle sont eux-mêmes pour ainsi dire planifiés selon des points de vue statistiques» (Adorno, 2003c : 296). Cf. : «l'idéologie utilise un nombre très limité d'idées et de trucs sans cesse répétés " (Adorno, 2003b:86).

19. «On ne risque nullement de surcharger les exemples choisis, car chacun est pars pro toto, et non seulement autorisé, mais contraint à revenir au système » (Adorno, 2003b : 85).

20. La notion de réalisme employée ici est synonyme de la fiction pour «adultes ", c'est-à-dire comportant des personnages crédibles et des décors qui ne sont pas visiblement en carton-pâte, comme dans la série Space Patrol (1950-55). Elle n'est pas forcément mesurable en termes de « réalisme social », peu compatible avec la récurrence, et avec le recours aux protagonistes aux qualités trop exceptionnelles pour être vaincus, ne serait-ce que de temps en temps. Une série comme Perry Mason (1957-66), où le célèbre avocat éponyme de la défense réussit à arracher l'aveu d'un témoin en plein procès à tous les coups, a pu être jugée réaliste pour les scènes de procédure juridique. Quelques exemples $d u$ "pseudo-réalisme» dans des séries récentes: le 
nombre ahurissant de marines trouvés assassinés dans le rayonnement de Washington DC., la juridiction de l'agence éponyme étant limité au personnel naval (NCIS) ; le nombre pas moins ahurissant de traîtres qui grouillent au sein de l'agence antiterroriste dans 24 heures chrono; le nombre incroyable dans les séries policières de personnes responsables (shérif, enseignant, fonctionnaire) coupables des pires bassesses; les accès informatiques et les tests scientifiques quasi instantanés (Les Experts). Cela n'a nullement empêché leur succès, d'où l'effet idéologique en creux : un certain « pseudo-réalisme » ne pose aucun problème en termes de crédibilité.

21. Le budget pour les décors et accessoires de la série quotidienne Captain Vidéo (1949-55) ne dépassait pas 25 \$ par semaine, ce qui se voit. (Tim Brooks, Earle Marsh, 1995 : 165).

22. Une série comme Mission impossible (1966-73), où l'équipe d'agents américains, au cours d'une intervention exigeant une organisation logistique inouïe, peut porter l'uniforme d'officier d'un pays étranger en parlant anglais, ainsi que le rôle d'aguicheuse donné au personnage féminin de l'équipe, serait impensable aujourd'hui. Autrement dit, il s'agit d'un irréalisme qui ne serait plus possible sur le plan idéologique.

23. cf. Adorno : «Loin de se mettre en mouvement et de s'assouplir, ce qui est figé et engourdi ne fait que s'endurcir. Les vocables de l'écriture par images sont des stéréotypes. Ils se justifient par les impératifs technologiques tels que celui qui consiste à produire en un temps minimum une quantité effrayante de matériau, ou à présenter sans tarder au spectateur les noms et les qualités des protagonistes... » (Adorno, 2003a : 69).

24. Il faudrait comparer cette intuition d'Adorno au concept (marxiste) de la figuration d'un projet idéologique, compris comme un assemblage stratégique d'éléments matériels. Ce concept, auquel je me suis déjà référé pour mon analyse des séries télévisées (Buxton, 2010), fut proposé par Pierre Macherey (1966) dans son analyse magnifique du roman de Jules Verne, L'Ile mystérieuse.

25. Le contexte ici est une discussion portant sur l'autonomie de l'art « authentique ». Admettons qu'Adorno n'a sûrement jamais envisagé de traiter les produits de la Kulturindustrie comme des œuvres d'art. Néanmoins, l'idée peut s'appliquer aussi à ces produits, à condition de remplacer la notion d'authenticité par celle de l'idéologie. Adorno précise que la télévision est « une sphère qui ignore l'autonomie esthétique » (Adorno, 2003b : 73).

26. À partir des années 1990, un nouveau format s'imposa : des épisodes de 41 minutes, avec 18 minutes d'annonces, afin de financer des séries deux fois plus chères en dollars constants depuis les années 1960 (aujourd'hui, trois fois plus chères), et dépendant des ventes à l'international pour rentrer dans les frais (les séries sont typiquement « louées » aux chaînes à $75 \%$ de leurs frais sur le marché américain). Voir David Buxton (2010 : 71).

27. Parlant au début des années 1960 de la série Bonanza, le critique littéraire John Cawelti a insisté sur sa pertinence contemporaine : «Le ranch des Cartwright est entouré de chicaneries, de violence et de trahison, presque comme l'harmonieuse banlieue de la classe moyenne américaine est menacée par les forces explosives d'une société en expansion. Mais la cohésion, la loyauté mutuelle et les qualités d'adaptation de la famille Cartwright se révèlent toujours capables de rejeter ou d'émousser le tranchant des forces d'invasion. »Cité in Rita Parks 1982 : 151.

28. C'est le moment d'apporter ma pierre à la notion adornienne de "stéréotype ». Les personnages dans les séries westerns relèvent plutôt d'archétypes renvoyant à l'idée d'une nature humaine universelle, passable de jugements moraux plus ou moins nuancés (le lâche, le droit dans ses bottes, le type qui s'est fourvoyé, mais qui a bon fond, le faux jeton, la garce intrigante). Les personnages dans les séries d'espionnage des années 1960 (Agents très spéciaux) relèvent plutôt de stéréotypes sociaux, passables d'un jugement plus directement idéologique. Ensuite, dans les séries policières qui dominent depuis les années 1970 se trouve un dosage subtil et parfois contre-intuitif de ces deux «types de personnalité », qui voit tel personnage immature, farceur et vantard (Tony DiNozzo de NCIS) agir en policier viril avec une droiture sans faille. Dans 
la mesure où une psychologie des personnages à l'américaine " passe » désormais dans le monde entier, même pour les sitcoms depuis les années 1990 (Friends), on peut parler objectivement d'une « américanisation » des consciences. Voir Buxton, 2010 : 15-22.

29. Stéphane Benassi situe l'origine de la série feuilletonnante dans ce qu'il appelle «la série de la quête » (Le Fugitif, 1963-67 ; Les Envahisseurs, 1967-68). Voir Benassi, 2000 : 85-92. Plus complexe, la série feuilletonnante marquait aussi l'émergence de l'écriture des scénarios en équipe, qui remplaçait l'ancien modèle d'écrivains indépendants et de "traitement " par un scénariste maison.

30. La formule de la série veut que Bauer traite en même temps une grave menace contre la sécurité nationale, et des problèmes personnels de son entourage, notamment dans les premières saisons les déboires de sa fille adolescente. Ensuite il doit faire torturer, au nom du devoir, l'exmari (britannique) de sa nouvelle amante (saison 4), ou (incroyablement) exécuter son patron sur les ordres d'un groupe terroriste pour sauver la ville de Los Angeles du pire (saison 3). Les malheurs du service du pays s'accumulent de saison en saison, culminant en trois ans sacrificiels de prison (tortures incluses) en Chine (entre les saisons 5 et 6 ).

31. Le feuilleton sentimental au rythme quotidien a pu distiller l'inflation des personnages dans une longue durée.

32. "Vérité " prononcée par un terroriste islamiste dans 24 heures chrono, saison 4, épisode 9 , 2004. On ne cherche pas à justifier les agissements du héros Bauer en termes autres que professionnels et (accessoirement) patriotiques. La tentative d'assassinat contre le candidat présidentiel David Palmer dans la première saison est motivée par la vengeance personnelle qui est aussi patriotique à sa façon: le Serbe André Drazen avait perdu des membres de sa famille dans les bombardements (illégaux selon la loi internationale) ordonnés par Palmer en sa qualité de président de la commission de la Défense au Sénat. La notion d'un haut-terreau moral qui s'impose à tous n'est plus tenable, chacun ayant droit maintenant à ses convictions.

33. Saison 4, épisode 9, 2005.

34. Saison 7, épisode 11, 2009. Ancien marine, capable d'une grande brutalité au service de son pays (torturer autrui, tuer de sang-froid), Bauer est aussi un tendre père et amant, intelligent et cultivé (il est titulaire d'une licence en lettres modernes de l'UCLA). Mais justement, le recours à la torture au service de son pays est illégal, et pose problème d'un point de vue politique, aux niveaux national et surtout international. Le fantôme de la guerre du Vietnam, et les crimes de guerre commis au cours d'un engagement perdu ne cessent de hanter l'imaginaire américain.

35. "Le fils prodigue », 1985. Cf. un épisode de Starsky et Hutch ("Nightlight », 1976) où les deux policiers éponymes laissent un trafiquant odieux dire que «celui qui a l'argent fait la loi », manifestant leur dégoût, mais sans le contredire.

36. Saison 6, épisode 22, « Biogenèse ", 1999.

37. Ces séries n'épargnent aucunement au spectateur la réalité biochimique de la décomposition d'un cadavre, et pour faire bonne mesure, on a recours aux images de synthèse pour donner à voir de l'intérieur du corps les dégâts provoqués par une trajectoire d'une balle ou d'une arme blanche.

38. Phrase récurrente entre avocats dans Suits : «Sors de mon bureau, espèce de merde ».

39. Adorno écrit dans son article de 1954 : «Ce qui est plus important, c'est [...] la disposition à voir des objets ordinaires comme si quelque mystère effrayant y était caché derrière. Une telle attitude semble aller de pair avec des illusions de masse comme la suspicion de dépravation omniprésente, de corruption et de conspiration » (Adorno, $1990: 241$ ).

40. «Le Procès", épisode 23. Cette série prend la relève de la peur maccarthyste que des Américains en apparence normale (qui aiment le baseball, qui lavent la voiture le samedi matin) pouvaient être en réalité des espions communistes, thème exploré ultérieurement The Americans (2013-). Il est intéressant que l'infortunée femme d'un extraterrestre « faussement » humain dans cet épisode doit raconter au tribunal les détails de son mariage : «il ne m'a pas touchée une fois. 
Il n'avait aucune pulsion humaine, aucun désir. Il avait quelque chose de froid, de presque inhumain ». Aucun désir pour les femmes, contre-définition normative qui vise par la bande l'homosexualité. L'exemple est proche de l'analyse classique de la paranoïa de Freud, notamment du cas du président Schreber, en termes d'homosexualité refoulée et du retournement (déformé) de l'affect. (Freud, 1954).

41. Saison 9, épisode 10, 2009.

42. Saison 1, épisode 12 (« Traque sans merci »), 2005.

43. Mention spéciale pour New York Unité spéciale (1999-) qui se limite aux crimes « monstrueux » (viols, pédophilie, exploitation monétaire de ceux-ci sur Internet, cf. « Meurtres sur Internet ", saison 1, épisode 18).

\section{INDEX}

Mots-clés : séries, télévision, industrie culturelle, Théorie critique, Theodor Adorno

\section{AUTEUR}

\section{DAVID BUXTON}

David Buxton est professeur en Sciences de l'information et de la communication à l'Université Paris Ouest-Nanterre-La Défense, où il participe du laboratoire HAR. 